\title{
AVALIAÇÃO DO HERBICIDA METSULFURON-METHYL NO CONTROLE DE PLANTAS DANINHAS EM ÁREA DE PRODUÇÃO DE SEMENTES DE PASTAGENS
}

\author{
Francisco de A. R. Pereira ${ }^{1}$, Amarildo J. Ornelas ${ }^{2}$ e Edison Hidalgo ${ }^{2}$ \\ ${ }^{1}$ Engenheiro Agrônomo, MSc, Pesquisador. EMPAER-MS. Caixa Postal 472. Campo Grande, MS 70031-902 frolimp@terra.com.br \\ ${ }^{2}$ Engenheiro Agrônomo, Desenvolvimento de Produtos. Du Pont do Brasil S.A. Caixa Postal 09. Paulínia, SP 13140-000
}

\begin{abstract}
RESUMO
Conduziu-se um experimento na Fazenda Bom Futuro, município de Rondonópolis, MT, em solo Areia Quartzosa, no ano agrícola 1999/2000, visando avaliar os efeitos do herbicida metsulfuron-methyl, aplicado em pós-emergência, no controle de plantas daninhas em campo de produção de sementes de Brachiaria brizantha (braquiária). Os tratamentos foram constituídos de metsulfuron-methyl a 3,0 g/ha em aplicação única, metsulfuron-methyl a 3,0 g/ha em duas épocas de aplicação, metsulfuronmethyl a 3,6 g/ha em aplicação única, metsulfuron-methyl a 3,6 g/ha em duas épocas de aplicação, 2,4-D a 288 g/ha (padrão) e testemunha. As plantas daninhas Tridax procumbens, Spermacoce latifolia e Chamaesyce hirta, foram suficientemente controladas através do metsulfuron-methyl a partir da dose de 3,0 g/ha, em aplicação única. Foram verificados sintomas leves de fitotoxicidade na braquiária, através dos tratamentos com aplicações em duas épocas e com o tratamento padrão 2,4-D. O rendimento de sementes de braquiária e o peso de 100 panículas foram superiores através dos tratamentos químicos, comparados à testemunha.
\end{abstract}

Palavras-chave: Brachiaria brizantha, 2,4-D, Tridax procumbens, Spermacoce latifolia, Chamaesyce hirta.

\section{ABSTRACT \\ Metsulfuron-methyl avaliation in the control of weeds in an área of pasture seed production}

An experiment was conducted at the Bom Futuro Farm, Rondonópolis, MT during 1999/2000 in a sandy soil, to determine the effects of metsulfuron-methyl on postemergence control of weeds in a field of Brachiaria brizantha seed production. Treatments were: metsulfuron-methyl at $3.0 \mathrm{~g} / \mathrm{ha}$ and at $3.6 \mathrm{~g} / \mathrm{ha}$ in one application or in two applications, 2,4-D at 288 g/ha (as a reference) and a check. Tridax procumbens, Spemacoce latifolia, Chamaesyce hirta were controlled by metsulfuron-methyl at 3.0 and $3.6 \mathrm{~g} / \mathrm{ha}$ in a sole application. It was observed slight injury to B. brizantha caused by the treatment with two applications of metsulfuron-methyl and by 2,4-D. B. brizantha seed production, weigth of 100 panicles were ligher in the treatments with herbicides.

Key words: Brachiaria brizantha, 2,4-D, Tridax procumbens, Spermacoce latifolia, Chamaesyce hirta. 


\section{INTRODUÇÃO}

A produção de sementes de forrageiras, visando atender à demanda de formação e/ou reforma de pastagens, vem gradativamente alcançando níveis tecnológicos adequados e coerentes com a importância da atividade, ou seja, a produção de sementes através de técnicas rudimentares, com baixo controle de qualidade, está aos poucos perdendo espaço, sobretudo considerando que uma boa semente, além de apresentar vigor e sanidade, não deve conter sementes de plantas daninhas. Em levantamento realizado por Mascarenhas et al. (1999) em pastagens de baixa produtividade na região nordeste do Pará, foram detectadas 118 espécies de plantas daninhas, abrangendo 34 famílias. Considerando que nesta região as roçadas, manual ou mecânica, constitui o método de controle de invasoras mais empregado, constata-se que as medidas adotadas não são suficientes para evitar-se o declínio do rendimento das pastagens provocado pelas plantas daninhas.

Para o controle químico de plantas daninhas em pastagens, como em qualquer cultura, é imprescindível que o herbicida apresente total seletividade à forrageira, permitindo-lhe desenvolvimento fenológico absolutamente normal. Rossi et al. (2000) desenvolveram pesquisas visando constatar a seletividade de herbicidas às pastagens formadas de capim-elefante e de "coastcross", concluindo que, dependendo do herbicida, pode haver redução no peso de matéria seca da forrageira. Dada a grande diversificação de espécies de plantas daninhas ocorrentes em pastagens, às vezes torna-se necessário a utilização de misturas de herbicidas, conforme resultados apresentados por Guimarães (2000) e Victoria Filho \& Ladeira Neto (2000).

A geração de subsídios para a produção de sementes que proporcione melhor qualidade e rendimento das pastagens, deve ser objeto de pesquisa, visando imprimir à atividade pecuária, maior poder de competitividade econômica.

Os objetivos do trabalho foram avaliar a eficiência e fitotoxicidade do herbicida metsulfuron-methyl, no controle de plantas daninhas, em campo de produção de sementes de Brachiaria brizantha, e gerar subsídios de praticabilidade agronômica na produção de sementes de forrageiras.

\section{MATERIAL E MÉTODOS}

O experimento foi instalado na Fazenda Bom Futuro, localizada no município de Rondonópolis, MT. O plantio da pastagem Brachiaria brizantha ocorreu no dia 17/09/99. As aplicações dos tratamentos, em pós-emergência, ocorreram nos dias 27/10/99 ( $1^{\mathrm{a}}$ aplicação) e no dia 03/12/99 ( $2^{\mathrm{a}}$ aplicação). Semeou-se a forrageira braquiária, espécie Brachiaria brizantha, com espaçamento de $68 \mathrm{~cm}$ entre linhas e $9 \mathrm{~cm}$ entre plantas. A área do ensaio foi adubada com $70 \mathrm{~kg} / \mathrm{ha}$ de
$\mathrm{KCL}$ e $120 \mathrm{~kg} /$ ha de MAP (mono hidratado de P) contendo $52 \%$ de $\mathrm{P}_{2} \mathrm{O}_{5}$.

O solo da área experimental era do tipo Areia Quartzosa , contendo $12,7 \%$ de argila, $81,4 \%$ de areia e 5,9\% de silte, $1,8 \%$ de matéria orgânica, e com relevo suave-ondulado, fase sob Cerrado.

Os tratamentos (Tabela 1), foram aplicados em pósemergência total em aplicação única e em duas épocas, abrangendo dois estádios de desenvolvimento da forrageira e das plantas daninhas (Tabela 2).

As parcelas experimentais mediam $3 \mathrm{~m} \times 6 \mathrm{~m}\left(18 \mathrm{~m}^{2}\right)$, com área útil de $10 \mathrm{~m}^{2}$ (2 m x $\left.5 \mathrm{~m}\right)$. O ensaio foi delineado em blocos ao acaso com 4 repetições e 6 tratamentos.

Para aplicação dos tratamentos utilizou-se um pulverizador costal de pressão constante, pressurizado por $\mathrm{CO}_{2}$, munido de uma barra com 4 bicos espaçados em $0,5 \mathrm{~m}$, com pontas XR 110.02. A pressão de trabalho foi de $2,1 \mathrm{~kg} / \mathrm{cm}^{2}$. O volume de calda aplicado foi de $2001 /$ ha . Durante a $1^{\text {a }}$ aplicação, em 27/10/99, das 07:05 às 07:50 horas, a temperatura média ambiente era de $27^{\circ} \mathrm{C}$, umidade relativa do ar $73 \%$ e ventos com velocidade média abaixo de $4 \mathrm{~km} /$ hora. Na $2^{\mathrm{a}}$ aplicação, em 03/12/99, das 08:15 às 09:00 horas, a temperatura média ambiente era de $26^{\circ} \mathrm{C}$, umidade relativa do ar $80 \%$ e ventos com velocidade média abaixo de $4 \mathrm{~km} /$ hora.

Realizaram-se avaliações de eficiência aos 15 e 30 dias após a aplicação dos tratamentos (DAT) ( $1^{\text {a }}$ aplicação), e aos $15,30,45$ DAT e na pré-colheita das sementes ( $2^{a}$ aplicação). A toxicidade à braquiária foi avaliada aos $7,10,15$ e $30 \mathrm{DAT}\left(1^{\mathrm{a}}\right.$ aplicação), e aos 7, 15, 30 e 45 DAT ( $2^{\mathrm{a}}$ aplicação). Empregouse o método de avaliação visual, através de uma escala de 0 a 100 , onde: $0=$ nenhuma injúria na planta e $100=$ morte total da planta. Para eficiência considerou-se a nota 80 como o padrão mínimo aceitável; para fitotoxicidade, considerou-se 40 a nota máxima aceitável, passível de recuperação da cultura, sem perspectivas de redução no rendimento econômico. Avaliou-se também o rendimento de sementes da braquiária, colhidas por amostragem na área útil das parcelas, sendo os dados transformados para $\mathrm{kg} / \mathrm{ha}$, peso de 100 e sementes (gramas) e peso de 100 panículas (gramas). Os dados foram analisados através da análise de variância e as médias comparadas pelo Teste de Tukey ao nível de $5 \%$ de probabilidade.

\section{RESULTADOS E DISCUSSÃO}

Os resultados das avaliações de fitotoxicidade sobre a braquiária mostraram que, nos tratamentos compostos por duas aplicações, a ocorrência de sintomas fitotóxicos foi mais acentuada, principalmente aos 7 e 15 dias após a segunda aplicação (Tabela 3). Porém constatou-se plena recuperação da forrageira e aos 45 dias após a segunda aplicação os sintomas eram imperceptíveis. 
Tabela 1. Descrição dos tratamentos. Rondonópolis-MT, 1999/2000.

\begin{tabular}{llcc}
\hline \multirow{2}{*}{ Produto comercial (p.c.) } & \multirow{2}{*}{ Nome comum } & \multicolumn{2}{c}{ Dose p.c. (g, l/ha) } \\
\cline { 3 - 4 } & metsulfuron-methyl & $\mathbf{1}^{\mathbf{a}}$ aplicação & $\mathbf{2}^{\mathbf{a}}$ aplicação \\
\hline Ally & metsulfuron-methyl & $5 \mathrm{~g}$ & - \\
Ally & metsulfuron-methyl & $6 \mathrm{~g}$ & $5 \mathrm{~g}$ \\
Ally & metsulfuron-methyl & $6 \mathrm{~g}$ & - \\
Ally & $2,4-D$ & 0,41 & $6 \mathrm{~g}$ \\
U-46 (Padrão) & - & - & $0,8 \mathrm{I}$ \\
Testemunha & & \\
\hline
\end{tabular}

Em todos os tratamentos com herbicidas adicionou-se Agral a $0,1 \% \mathrm{v} / \mathrm{v}$.

Tabela 2. Características das plantas daninhas avaliadas. Rondonópolis-MT, 1999/2000.

\begin{tabular}{|c|c|c|}
\hline \multirow{2}{*}{ Espécie } & \multicolumn{2}{|c|}{ Estádio/Densidade (plantas/m²) } \\
\hline & $1^{\mathrm{a}}$ Aplicação $^{1}$ & $2^{a}$ Aplicação ${ }^{2}$ \\
\hline Tridax procumbens & 4 folhas $/ 286 \mathrm{pl} / \mathrm{m}^{2}$ & em florescimento $/ 103 \mathrm{pl} / \mathrm{m}^{2}$ \\
\hline Spermacoce latifolia & 5 folhas/ $4 \mathrm{pl} / \mathrm{m}^{2}$ & início florescimento/4 pl/m² \\
\hline Chamaesyce hirta & 5 folhas/ $11 \mathrm{pl} / \mathrm{m}^{2}$ & em florescimento $/ 13 \mathrm{pl} / \mathrm{m}^{2}$ \\
\hline
\end{tabular}

${ }^{1} \mathrm{Na} 1^{\text {a }}$ aplicação a Brachiaria brizantha encontrava-se com 4 perfilhos, em média.

${ }^{2} \mathrm{Na} 2^{\mathrm{a}}$ aplicação a B. brizantha encontrava-se em início de alongamento.

Tabela 3. Média de toxicidade (\%) à Brachiaria brizantha. Rondonópolis-MT, 1999/2000.

\begin{tabular}{|c|c|c|c|c|c|c|c|c|c|c|}
\hline \multirow{2}{*}{ Tratamento $^{1}$} & \multirow{2}{*}{$\begin{array}{c}1^{\mathrm{a}} \text { aplicação } \\
(\mathrm{g} / \mathrm{ha})\end{array}$} & \multirow{2}{*}{$\begin{array}{c}2^{\mathrm{a} a p l i c a c ̧ a ̃ o ~} \\
\text { (g/ha) }\end{array}$} & \multicolumn{4}{|c|}{ Dias após a $1^{\text {a }}$ aplicação } & \multicolumn{4}{|c|}{ Dias após a $2^{\mathrm{a}}$ aplicação } \\
\hline & & & 07 & 10 & 15 & 30 & 07 & 15 & 30 & 45 \\
\hline Metsulfuron-methyl & 3,0 & - & 2 & 9 & 0 & 0 & 0 & 0 & 0 & 0 \\
\hline Metsulfuron-methyl & 3,0 & 3,0 & 2 & 9 & 0 & 0 & 25 & 24 & 17 & 0 \\
\hline Metsulfuron-methyl & 3,6 & - & 4 & 10 & 0 & 0 & 0 & 0 & 0 & 0 \\
\hline Metsulfuron-methyl & 3,6 & 3,6 & 4 & 10 & 0 & 0 & 33 & 29 & 18 & 0 \\
\hline 2,4-D & 288 & 288 & 9 & 23 & 14 & 16 & 26 & 29 & 20 & 0 \\
\hline Testemunha & - & - & 0 & 0 & 0 & 0 & 0 & 0 & 0 & 0 \\
\hline
\end{tabular}

Foi aplicado Agral $(0,1 \% \mathrm{v} / \mathrm{v}) \mathrm{em}$ todos os tratamentos com herbicidas.

Até os 45 dias após a segunda aplicação o controle das plantas daninhas foi exercido exclusivamente pela ação dos herbicidas. A partir dos 45 dias após a segunda aplicação, a integração entre o controle químico e o controle cultural exercido pela braquiária, proporcionou um resultado final excelente em todos os tratamentos químicos, conforme pode-se constatar nas avaliações de eficiência (Tabelas 4, 5 e 6). Os tratamentos com metsulfuron-methyl apresentaram melhor controle que o tratamento padrão $(2,4$ D), sobre a espécie Chamaesyce hirta (Tabela 6).
Os resultados de rendimento de sementes (Tabela 7), foram semelhantes entre os tratamentos químicos, diferenciando-se estatisticamente apenas com relação à testemunha.

Os resultados de peso de 100 sementes não apresentaram diferença estatística entre si (Tabela 7). Nas avaliações do peso de 100 panículas, constatou-se que os melhores resultados foram obtidos através dos tratamentos onde ocorreram a segunda aplicação de herbicidas, seguidos dos tratamentos com uma aplicação e todos diferenciados da testemunha (Tabela 7). 
Tabela 4. Média de controle (\%) de Tridax procumbens. Rondonópolis-MT, 1999/2000.

\begin{tabular}{|c|c|c|c|c|c|c|c|c|}
\hline \multirow{2}{*}{ Tratamento ${ }^{1}$} & \multirow{2}{*}{$\begin{array}{c}1 \text { a aplicação } \\
\text { (g/ha) }\end{array}$} & \multirow{2}{*}{$\begin{array}{c}2^{\mathrm{a} a p l i c a c ̧ a ̃ o ~} \\
\text { (g/ha) }\end{array}$} & \multicolumn{2}{|c|}{ Dias após a $1^{\text {a }}$ aplicação } & \multicolumn{4}{|c|}{ Dias após a $2^{\mathrm{a}}$ aplicação } \\
\hline & & & 15 & 30 & 15 & 30 & 45 & Pré \\
\hline Metsulfuron-methyl & 3,0 & - & 74 & 70 & 69 & 67 & 65 & 85 \\
\hline Metsulfuron-methyl & 3,0 & 3,0 & 70 & 70 & 87 & 90 & 93 & 100 \\
\hline Metsulfuron-methyl & 3,6 & - & 78 & 71 & 76 & 73 & 70 & 87 \\
\hline Metsulfuron-methyl & 3,6 & 3,6 & 78 & 74 & 91 & 92 & 95 & 100 \\
\hline 2,4-D & 288 & 288 & 55 & 60 & 98 & 100 & 100 & 100 \\
\hline Testemunha & - & - & 0 & 0 & 0 & 0 & 0 & 0 \\
\hline
\end{tabular}

${ }^{1}$ Foi aplicado Agral $(0,1 \% \mathrm{v} / \mathrm{v})$ em todos os tratamentos com herbicidas.

Tabela 5. Média de controle (\%) de Spermacocea latifolia. Rondonópolis-MT, 1999/2000.

\begin{tabular}{|c|c|c|c|c|c|c|c|c|}
\hline \multirow{2}{*}{ Tratamento $^{1}$} & \multirow{2}{*}{$\begin{array}{c}\text { 1'aplicação } \\
\text { (g/ha) }\end{array}$} & \multirow{2}{*}{$\begin{array}{c}2^{\text {a aplicação }} \\
\text { (g/ha) }\end{array}$} & \multicolumn{2}{|c|}{ Dias após a $1^{\mathrm{a}}$ aplicação } & \multicolumn{4}{|c|}{ Dias após a $2^{\mathrm{a}}$ aplicação } \\
\hline & & & 15 & 30 & 15 & 30 & 45 & Pré \\
\hline Metsulfuron-methyl & 3,0 & - & 69 & 59 & 59 & 60 & 75 & 80 \\
\hline Metsulfuron-methyl & 3,0 & 3,0 & 69 & 58 & 77 & 77 & 84 & 88 \\
\hline Metsulfuron-methyl & 3,6 & - & 72 & 64 & 68 & 69 & 76 & 80 \\
\hline Metsulfuron-methyl & 3,6 & 3,6 & 83 & 63 & 81 & 83 & 87 & 89 \\
\hline $2,4-\mathrm{D}$ & 288 & 288 & 35 & 23 & 75 & 77 & 85 & 89 \\
\hline Testemunha & - & - & 0 & 0 & 0 & 0 & 0 & 0 \\
\hline
\end{tabular}

${ }^{1}$ Foi aplicado Agral $(0,1 \% \mathrm{v} / \mathrm{v})$ em todos os tratamentos com herbicidas.

Tabela 6. Média de controle (\%) de Chamaesyce hirta. Rondonópolis-MT, 1999/2000.

\begin{tabular}{|c|c|c|c|c|c|c|c|c|}
\hline \multirow{2}{*}{ Tratamento $^{1}$} & \multirow{2}{*}{$\begin{array}{c}1 \text { a aplicação } \\
\text { (g/ha) }\end{array}$} & \multirow{2}{*}{$\begin{array}{c}2^{\text {a aplicação }} \\
\text { (g/ha) }\end{array}$} & \multicolumn{2}{|c|}{ Dias após a $1^{a}$ aplicação } & \multicolumn{4}{|c|}{ Dias após a $2^{\mathrm{a}}$ aplicação } \\
\hline & & & 15 & 30 & 15 & 30 & 45 & Pré \\
\hline Metsulfuron-methyl & 3,0 & - & 96 & 95 & 83 & 84 & 87 & 95 \\
\hline Metsulfuron-methyl & 3,0 & 3,0 & 93 & 93 & 98 & 98 & 100 & 100 \\
\hline Metsulfuron-methyl & 3,6 & - & 91 & 89 & 86 & 85 & 89 & 97 \\
\hline Metsulfuron-methyl & 3,6 & 3,6 & 90 & 94 & 100 & 100 & 100 & 100 \\
\hline $2,4-\mathrm{D}$ & 288 & 288 & 40 & 15 & 60 & 58 & 63 & 74 \\
\hline Testemunha & - & - & 0 & 0 & 0 & 0 & 0 & 0 \\
\hline
\end{tabular}

${ }^{1}$ Foi aplicado Agral $(0,1 \% \mathrm{v} / \mathrm{v})$ em todos os tratamentos com herbicidas.

\section{CONCLUSÕES}

Considerando o controle satisfatório exercido sobre as plantas daninhas, a seletividade sobre a forrageira e a preservação do seu do potencial produtivo, conclui-se:

1. O herbicida metsulfuron-methyl (Ally), em dose única de $3 \mathrm{~g} / \mathrm{ha}+$ Agral 0,1\% v/v, aplicado em pós-emergência durante o perfilhamento de Brachiaria brizantha, controla satisfatoriamente as plantas daninhas Tridax procumbens (4 folhas), Spermacoce latifolia (5 folhas) e Chamaesyce hirta (5 folhas) e não provoca nenhuma injúria à $B$. brizantha.

2. Os tratamentos químicos avaliados apresentam praticabilidade agronômica em área de produção de sementes de B. brizantha, infestada com T.procumbens, $S$. latifolia e C. hirta. 
Tabela 7. Rendimento de sementes de Brachiaria brizantha, peso de 100 sementes e peso de 100 panículas. RondonópolisMT, 1999/2000.

\begin{tabular}{|c|c|c|c|c|c|}
\hline Tratamento ${ }^{1}$ & $\begin{array}{c}1^{\text {a }} \text { aplicação } \\
\text { (g/ha) }\end{array}$ & $\begin{array}{c}2^{\text {a aplicação }} \\
\text { (g/ha) }\end{array}$ & $\begin{array}{c}\text { Rendimento } \\
\text { (kg/ha) }\end{array}$ & $\begin{array}{l}\text { Peso de } 100 \\
\text { sementes (g) }\end{array}$ & $\begin{array}{c}\text { Peso de } 100 \\
\text { panículas (g) }\end{array}$ \\
\hline Metsulfuron-methyl & 3,0 & - & $2675 \mathrm{a}$ & $0,55 \mathrm{a}$ & $44,5 \mathrm{ab}$ \\
\hline Metsulfuron-methyl & 3,0 & 3,0 & $2530 \mathrm{a}$ & $0,50 \mathrm{a}$ & $52,0 \mathrm{a}$ \\
\hline Metsulfuron-methyl & 3,6 & - & $2780 \mathrm{a}$ & $0,47 \mathrm{a}$ & $45,5 \mathrm{ab}$ \\
\hline Metsulfuron-methyl & 3,6 & 3,6 & $2800 \mathrm{a}$ & $0,47 \mathrm{a}$ & $50,7 \mathrm{a}$ \\
\hline $2,4-\mathrm{D}$ & 288 & 288 & $2595 \mathrm{a}$ & $0,47 \mathrm{a}$ & $44,0 \mathrm{ab}$ \\
\hline Testemunha & - & - & $2010 \mathrm{~b}$ & $0,45 \mathrm{a}$ & $41,2 \mathrm{~b}$ \\
\hline C.V. $(\%)$ & & & 23,7 & $15,7 \mathrm{a}$ & 7,5 \\
\hline
\end{tabular}

${ }^{1}$ Foi aplicado Agral $(0,1 \% \mathrm{v} / \mathrm{v})$ em todos os tratamentos com herbicidas.

\section{LITERATURA CITADA}

GUIMARÃES, S. C. Controle de Senna obtusifolia e Hyptis suaveolens, em área de reforma de pastagem, com a mistura de picloram potássico e metsulfuron-methyl. In: CONGRESSO BRASILEIRO DA CIÊNCIA DAS PLANTAS DANINHAS, 22, 2000. RESUMOS... Foz do Iguaçu:SBCPD, 2000.p. 361.

MASCARENHAS, R. E. B.; MODESTO JUNIOR, M. S.; DUTRA, S.; SOUZA FILHO, A. P. S.; TEIXEIRA NETO, J. F. Plantas daninhas de uma pastagem cultivada de baixa produtividade no nordeste paraense. Planta Daninha, v. 17, n. 3, p. 399-418, 1999.
ROSSI, P. ; MACIEL, C. D. G.; MARTINS, D.; COSTA, C. Seletividade de herbicidas aplicados em pós-emergência em capim-elefante (Pennisetum purpureum) e Cynodon dactylon cv. Coastcross. In: CONGRESSO BRASILEIRO DA CIÊNCIA DAS PLANTAS DANINHAS, 22, 2000. RESUMOS... Foz do Iguaçu: SBCPD, 2000. p. 357-58.

VICTORIA FILHO, R.; LADEIRA NETO, A. Efeito de misturas de fluroxypyr + picloram no controle do leiteiro (Peschiera fuchsiaefolia) em pastagens com aplicação foliar. In: CONGRESSO BRASILEIRO DA CIÊNCIA DAS PLANTAS DANINHAS, 22, 2000. RESUMOS... Foz do Iguaçu: SBCPD, 2000.p. 360. 
Revista Brasileira de Herbicidas v.1, n.2, 2000 